\title{
On the boundary value problems for quasiconformal functions in the plane
}

\author{
Vladimir Gutlyanskit, Vladimir Ryazanov, \\ ARTEM YeFIMUSHKIN
}

\begin{abstract}
Generalized solvability of the classical boundary value problems for analytic and quasiconformal functions in arbitrary Jordan domains with boundary data that are measurable with respect to the logarithmic capacity is established. Moreover, it is shown that the spaces of the found solutions have the infinite dimension. Finally, some applications to the boundary value problems for A-harmonic functions are given.
\end{abstract}

2010 MSC. 31A05, 31A20, 31A25, 31B25, 35Q15, 30E25, 31C05, 35F45.

Key words and phrases. Dirichlet, Hilbert, Riemann, Neumann, Poincare problems, analytic functions, quasiconformal functions, Beltrami equations, A-harmonic functions, nonlinear problems.

\section{Introduction}

The classical boundary value problems of the theory of analytic functions, such as the Dirichlet, Hilbert, Riemann, Neumann and Poincare problems, play a fundamental role in contemporary analysis and its applications to actual problems of mathematical physics. Note that these boundary value problems are closely interconnected, see e.g. monographs $[9,21,29]$, and also recent works [6, 25-28].

In this paper we continue the development of the theory of the above boundary value problems for analytic functions in arbitrary Jordan domains with measurable boundary data and extend the theory to the more general class of quasiconformal functions. The latter class of functions is generated by the regular solutions of the well-known Beltrami equation, see e.g. [18]. Recall that quasiconformal functions form just the class of mappings with bounded distortion by Reshetnyak, see e.g. [24], or, equivalently, the class of quasiregular mappings, see e.g. [19].

Received 02.09.2015 
Let $D$ be a domain in the complex plane $\mathbb{C}$ and $\mu: D \rightarrow \mathbb{C}$ be a measurable function with $|\mu(z)|<1$ a.e. Recall that a partial differential equation

$$
f_{\bar{z}}=\mu(z) \cdot f_{z}
$$

where $f_{\bar{z}}=\bar{\partial} f=\left(f_{x}+i f_{y}\right) / 2, f_{z}=\partial f=\left(f_{x}-i f_{y}\right) / 2, z=x+i y, f_{x}$ and $f_{y}$ are partial derivatives of the function $f$ in $x$ and $y$, respectively, is said to be a Beltrami equation. The Beltrami equation (1.1) is said to be nondegenerate if $\|\mu\|_{\infty}<1$.

Note that analytic functions satisfy (1.1) with $\mu(z) \equiv 0$. Recall also that a regular solution of the Beltrami equation is a continuous, discrete and open mapping $f: D \rightarrow \mathbb{C}$ of the Sobolev class $W_{\text {loc }}^{1,1}$ satisfying (1.1) a.e. Note that, in the case of nondegenerate Beltrami equations, a regular solution $f$ belongs to class $W_{\text {loc }}^{1, p}$ for some $p>2$ and, moreover, its Jacobian $J_{f}(z) \neq 0$ for almost all $z \in D$, and it is called a quasiconformal function, see e.g. Chapter VI in [18]. Moreover, $f$ is called a quasiconformal mapping if in addition it is a homeomorphism.

It is clear that if we start to consider boundary value problems with measurable boundary data, then the requests on the existence of the limits at all points $\zeta \in \partial D$ and along all paths terminating in $\zeta$ (as well as the conception of the index) lose any sense. Thus, the notion of solutions of such boundary value problems should be extended. The nontangential limits from the function theory of a complex variable were a suitable tool, see e.g. [6] and [25]. In [28], it was proposed an alternative approach admitting tangential limits for analytic functions. At present paper, we use this approach to the Beltrami equations.

One of the relevant problems concerns to the measurement of sets on boundaries of domains. In this connection, note that the sets of the length measure zero as well as of the harmonic measure zero are invariant under conformal mappings, however, they are not invariant under quasiconformal mappings. The latter follows, e.g., from the famous Ahlfors-Beurling example of quasisymmetric mappings of the real axis that are not absolutely continuous, see [2]. Hence we are forced to apply here the so-called absolute harmonic measure by Nevanlinna, in other words, logarithmic capacity whose zero sets are invariant under quasiconformal mappings, see e.g. [22].

\section{Definitions and preliminary remarks}

Let $\mathbb{D}=\{z \in \mathbb{C}:|z|<1\}$ be the unit disk in the complex plane $\mathbb{C}$. By the well-known Priwalow uniqueness theorem analytic functions in $\mathbb{D}$ coincide if they have the equal boundary values along all nontangential 
paths to a set $E$ of points in $\partial \mathbb{D}$ of a positive measure, see e.g. Theorem IV.2.5 in [23]. The theorem is valid also for analytic functions in Jordan domains with rectifiable boundaries, see e.g. Section IV.2.6 in [23].

However, examples of Lusin and Priwalow show that there exist nontrivial analytic functions in $\mathbb{D}$ whose radial boundary values are equal to zero on sets $E \subseteq \partial \mathbb{D}$ of a positive measure, see e.g. Section IV.5 in [23]. Simultaneously, by Theorem IV.6.2 in [23] of Lusin and Priwalow the uniqueness result is valid if $E$ is of the second category. Theorem 1 in [4] demonstrates that the latter condition is necessary.

Recall Baire's terminology for categories of sets and functions. Namely, given a topological space $X$, a set $E \subseteq X$ is of first category if it can be written as a countable union of nowhere dense sets, and is of second category if $E$ is not of first category. Also, given topological spaces $X$ and $X_{*}, f: X \rightarrow X_{*}$ is said to be a function of Baire's class 1 if $f^{-1}(U)$ for every open set $U$ in $X_{*}$ is an $F_{\sigma}$ set in $X$ where an $F_{\sigma}$ set is the union of a sequence of closed sets.

Given a Jordan curve $C$ in $\mathbb{C}$, we say that a family of Jordan arcs $\left\{J_{\zeta}\right\}_{\zeta \in C}$ is of class $\mathcal{B S}$ (of the Bagemihl-Seidel class), cf. [4], 740-741, if all $J_{\zeta}$ lie in a ring $\mathfrak{R}$ generated by $C$ and a Jordan curve $C_{*}$ in $\mathbb{C}$, $C_{*} \cap C=\varnothing, J_{\zeta}$ is joining $C_{*}$ and $\zeta \in C$, every $z \in \mathfrak{R}$ belongs to a single arc $J_{\zeta}$, and for a sequence of mutually disjoint Jordan curves $C_{n}$ in $\mathfrak{R}$ such that $C_{n} \rightarrow C$ as $n \rightarrow \infty, J_{\zeta} \cap C_{n}$ consists of a single point for each $\zeta \in C$ and $n=1,2, \ldots$

In particular, a family of Jordan arcs $\left\{J_{\zeta}\right\}_{\zeta \in C}$ is of class $\mathcal{B S}$ if $J_{\zeta}$ are induced by an isotopy of $C$. For instance, every curvilinear ring $\mathfrak{R}$ one of whose boundary component is $C$ can be mapped with a conformal mapping $g$ onto a circular ring $R$ and the inverse mapping $g^{-1}: R \rightarrow \mathfrak{R}$ maps radial lines in $R$ onto suitable Jordan arcs $J_{\zeta}$ and centered circles in $R$ onto Jordan curves giving the corresponding isotopy of $C$ to other boundary component of $\mathfrak{R}$.

Now, if $\Omega \subset \mathbb{C}$ is an open set bounded by a finite collection of mutually disjoint Jordan curves, then we say that a family of Jordan arcs $\left\{J_{\zeta}\right\}_{\zeta \in \partial \Omega}$ is of class $\mathcal{B S}$ if its restriction to each component of $\partial \Omega$ is so.

Theorem 1 in [4] can be written in the following way, see footnote 9 in [4].

Proposition 1. Let $D$ be a bounded domain in $\mathbb{C}$ whose boundary consists of a finite number of mutually disjoint Jordan curves and let $\left\{\gamma_{\zeta}\right\}_{\zeta \in \partial D}$ be a family of Jordan arcs of class $\mathcal{B S}$ in $D$.

Suppose $M$ is an $F_{\sigma}$ set of first category on $\partial D$ and $\Phi(\zeta)$ is a complexvalued function of Baire's class 1 on $M$. Then there is a nonconstant single-valued analytic function $f: D \rightarrow \mathbb{C}$ such that, for all $\zeta \in M$, 
along $\gamma_{\zeta}$

$$
\lim _{z \rightarrow \zeta} f(z)=\Phi(\zeta)
$$

On this basis, in the case of domains $D$ whose boundaries consist of rectifiable Jordan curves, it was formulated Theorem 2 in [4] on the existence of analytic functions $f: D \rightarrow \mathbb{C}$ such that (2.1) holds a.e. on $\partial D$ with respect to the natural parameter for each prescribed measurable function $\Phi: \partial D \rightarrow \mathbb{C}$.

\section{On the Dirichlet problem for analytic functions}

We need for our goals the following statement on analytic functions with prescribed boundary values that is similar to Theorem 2 in [4] but formulated in terms of logarithmic capacity instead of the natural parameter, see definitions, notations and comments in [6].

Theorem 1. Let $D$ be a bounded domain in $\mathbb{C}$ whose boundary consists of a finite number of mutually disjoint Jordan curves and let a function $\Phi: \partial D \rightarrow \mathbb{C}$ be measurable with respect to the logarithmic capacity.

Suppose that $\left\{\gamma_{\zeta}\right\}_{\zeta \in \partial D}$ is a family of Jordan arcs of class $\mathcal{B S}$ in $D$. Then there is a nonconstant single-valued analytic function $f: D \rightarrow$ $\mathbb{C}$ such that (2.1) holds along $\gamma_{\zeta}$ for a.e. $\zeta \in \partial D$ with respect to the logarithmic capacity.

Proof. Note first of all that $\mathcal{C}:=C(\partial D)<\infty$ because $\partial D$ is bounded and Borel, even compact, and show that there is a sigma-compact set $S$ in $\partial D$ of first category such that $C(S)=\mathcal{C}$. More precisely, $S$ will be the union of a sequence of sets $S_{m}$ in $\partial D$ of the Cantor type that are nowhere dense in $\partial D$.

Namely, $S_{m}$ is constructed in the following way. First we remove an open arc $A_{1}$ in $\partial D$ of the logarithmic capacity $2^{-m} \mathcal{C}$ and one more open arc $A_{2}$ in $\partial D \backslash A_{1}$ of the logarithmic capacity $2^{-(m+1)} \mathcal{C}$ such that $\partial D \backslash\left(A_{1} \cup A_{2}\right)$ consists of 2 segments of $\partial D$ with the equal logarithmic capacity. Then we remove a union $A_{3}$ of 2 open arcs in each these segments of the total logarithmic capacity $2^{-(m+2)} \mathcal{C}$ such that new 4 segments in $\partial D \backslash\left(A_{1} \cup A_{2} \cup A_{3}\right)$ have the equal logarithmic capacity. Repeating by induction this construction, we obtain the compact sets $S_{m}=\partial D \backslash \bigcup_{m=1}^{\infty} A_{m}$ with the logarithmic capacity $\left(1-2^{-(m-1)}\right) \cdot \mathcal{C} \rightarrow \mathcal{C}$ as $m \rightarrow \infty$.

Note also that the logarithmic capacity is Borel's regular measure as well as Radon's measure in the sense of points 2.2.3 and 2.2.5 in [7], 
correspondingly, see Section 2 in [6]. Hence the Lusin theorem holds for the logarithmic capacity on $\mathbb{C}$, see Theorem 2.3.5 in [7].

By the Lusin theorem one can find a sequence of compacta $K_{n}$ in $S$ with $C\left(S \backslash K_{n}\right)<2^{-n}$ such that $\left.\Phi\right|_{K_{n}}$ is continuous for each $n=1,2, \ldots$, i.e., for every open set $U \subseteq \mathbb{C}, W_{n}:=\left.\Phi\right|_{K_{n}} ^{-1}(U)=V_{n} \cap S$ for some open set $V_{n}$ in $\mathbb{C}$, and $W_{n}$ is sigma-compact because $V_{n}$ and $S$ are sigma-compact. Consequently, $W:=\left.\Phi\right|_{K} ^{-1}(U)=\bigcup_{n=1}^{\infty} W_{n}$ is also sigma-compact where $K=\bigcup_{n=1}^{\infty} K_{n}$. Hence the restriction of $\Phi$ on the set $K$ is a function of Baire's class 1 . Finally, note that by the construction $C(Z)=0$ where $Z=\partial D \backslash K=(\partial D \backslash S) \cup(S \backslash K)$ and

$$
K=\bigcup_{m, n=1}^{\infty}\left(S_{m} \cap K_{n}\right)
$$

where each set $E_{m, n}:=S_{m} \cap K_{n}, m, n=1,2, \ldots$, is nowhere dense in $\partial D$.

Thus, the conclusion of Theorem 1 follows from Proposition 1.

Corollary 1. Let $D$ be a bounded domain in $\mathbb{C}$ whose boundary consists of a finite number of mutually disjoint Jordan curves and let a function $\varphi: \partial D \rightarrow \mathbb{R}$ be measurable with respect to the logarithmic capacity.

Suppose that $\left\{\gamma_{\zeta}\right\}_{\zeta \in \partial D}$ is a family of Jordan arcs of class $\mathcal{B S}$ in $D$. Then there is a harmonic function $u: D \rightarrow \mathbb{R}$ such that

$$
\lim _{z \rightarrow \zeta} u(z)=\varphi(\zeta)
$$

along $\gamma_{\zeta}$ for a.e. $\zeta \in \partial D$ with respect to the logarithmic capacity.

Corollary 2. Let $D$ be a Jordan domain in $\mathbb{C}$ and a function $\Phi: \partial D \rightarrow \mathbb{C}$ be measurable with respect to the logarithmic capacity.

Suppose that $\left\{\gamma_{\zeta}\right\}_{\zeta \in \partial D}$ is a family of Jordan arcs of class $\mathcal{B S}$ in $D$. Then there is a harmonic function $u: D \rightarrow \mathbb{R}$ such that

$$
\lim _{z \rightarrow \zeta} \nabla u(z)=\Phi(\zeta)
$$

along $\gamma_{\zeta}$ for a.e. $\zeta \in \partial D$ with respect to the logarithmic capacity.

Here we use the complex notation for the gradient $\nabla u:=u_{x}+i \cdot u_{y}$.

Proof. Indeed, by Theorem 1 there is a single-valued analytic function $f: D \rightarrow \mathbb{R}$ such that

$$
\lim _{z \rightarrow \zeta} f(z)=\overline{\Phi(\zeta)}
$$


along $\gamma_{\zeta}$ for a.e. $\zeta \in \partial D$ with respect to the logarithmic capacity. Then any indefinite integral $F$ of $f$ is also a single-valued analytic function in the simply connected domain $D$ and the harmonic functions $u=\operatorname{Re} F$ and $v=\operatorname{Im} F$ satisfy the Cauchy-Riemann system $v_{x}=-u_{y}$ and $v_{y}=$ $u_{x}$. Hence

$$
f=F^{\prime}=F_{x}=u_{x}+i \cdot v_{x}=u_{x}-i \cdot u_{y}=\overline{\nabla u} .
$$

Thus, (3.2) follows from (3.3) and, consequently, $u$ is the required function.

Corollary 3. Let a function $\Phi: \partial \mathbb{D} \rightarrow \mathbb{C}$ be measurable with respect to the logarithmic capacity. Then there is a harmonic function $u: \mathbb{D} \rightarrow \mathbb{R}$ such that (3.2) holds along radial lines for a.e. $\zeta \in \partial \mathbb{D}$ with respect to the logarithmic capacity.

\section{On the Dirichlet problem for quasiconformal functions}

We show that quasiconformal functions are able to take arbitrary measurable values along prescribed families of Jordan arcs terminating at the boundary.

Theorem 2. Let $D$ be a bounded domain in $\mathbb{C}$ whose boundary consists of a finite number of mutually disjoint Jordan curves, $\mu: D \rightarrow \mathbb{C}$ be a Lebesgue measurable function with $\|\mu\|_{\infty}<1$, and let a function $\Phi$ : $\partial D \rightarrow \mathbb{C}$ be measurable with respect to the logarithmic capacity.

Suppose that $\left\{\gamma_{\zeta}\right\}_{\zeta \in \partial D}$ is a family of Jordan arcs of class $\mathcal{B S}$ in $D$. Then the Beltrami equation (1.1) has a regular solution $f: D \rightarrow \mathbb{C}$ such that (2.1) holds along $\gamma_{\zeta}$ for a.e. $\zeta \in \partial D$ with respect to the logarithmic capacity.

Proof. Extending $\mu$ by zero everywhere outside of $D$, we obtain the existence of a quasiconformal mapping $h: \mathbb{C} \rightarrow \mathbb{C}$ a.e. satisfying the Beltrami equation (1.1) with the given $\mu$, see e.g. Theorem V.B.3 in [1]. Setting $D_{*}=h(D)$ and $\Gamma_{\xi}=h\left(\gamma_{h^{-1}(\xi)}\right), \xi \in \partial D_{*}$, we see that $\left\{\Gamma_{\xi}\right\}_{\xi \in \partial D_{*}}$ is a family of Jordan arcs of class $\mathcal{B S}$ in $D_{*}$.

The logarithmic capacity of a set coincides with its transfinite diameter, see e.g. [8] and the point 110 in [22]. Hence the mappings $h$ and $h^{-1}$ transform sets of logarithmic capacity zero on $\partial D$ into sets of logarithmic capacity zero on $\partial D_{*}$ and vice versa because these quasiconformal mappings are continuous by Hölder on $\partial D$ and $\partial D_{*}$ correspondingly, see e.g. Theorem II.4.3 in [18]. 
Further, the function $\varphi=\Phi \circ h^{-1}$ is measurable with respect to logarithmic capacity. Indeed, under this mapping measurable sets with respect to logarithmic capacity are transformed into measurable sets with respect to logarithmic capacity because such a set can be represented as the union of a sigma-compactum and a set of logarithmic capacity zero. On the other hand, compacta under continuous mappings are transformed into compacta and compacta are measurable sets with respect to logarithmic capacity.

By Theorem 1 there is an analytic function $F: D_{*} \rightarrow \mathbb{C}$ such that

$$
\lim _{w \rightarrow \xi} F(w)=\varphi(\xi)
$$

holds along $\Gamma_{\xi}$ for a.e. $\xi \in \partial D_{*}$ with respect to logarithmic capacity. Thus, by the arguments given above $f:=F \circ h$ is the desired solution of (1.1).

\section{On Riemann-Hilbert problem for analytic functions}

Recall that the classical setting of the Hilbert (Riemann-Hilbert) boundary value problem is to find analytic functions $f$ in a domain $D \subset \mathbb{C}$ bounded by a rectifiable Jordan curve with the boundary condition

$$
\lim _{z \rightarrow \zeta} \operatorname{Re}\{\overline{\lambda(\zeta)} \cdot f(z)\}=\varphi(\zeta) \quad \forall \zeta \in \partial D
$$

where functions $\lambda$ and $\varphi$ were continuously differentiable with respect to the natural parameter $s$ on $\partial D$ and, moreover, $|\lambda| \neq 0$ everywhere on $\partial D$. Hence without loss of generality one can assume that $|\lambda| \equiv 1$ on $\partial D$.

Theorem 3. Let $D$ be a bounded domain in $\mathbb{C}$ whose boundary consists of a finite number of mutually disjoint Jordan curves, and let $\lambda: \partial D \rightarrow \mathbb{C}$, $|\lambda(\zeta)| \equiv 1, \varphi: \partial D \rightarrow \mathbb{R}$ and $\psi: \partial D \rightarrow \mathbb{R}$ be measurable functions with respect to the logarithmic capacity.

Suppose that $\left\{\gamma_{\zeta}\right\}_{\zeta \in \partial D}$ is a family of Jordan arcs of class $\mathcal{B S}$ in $D$. Then there is a nonconstant single-valued analytic function $f: D \rightarrow \mathbb{C}$ such that

$$
\begin{aligned}
& \lim _{z \rightarrow \zeta} \operatorname{Re}\{\overline{\lambda(\zeta)} \cdot f(z)\}=\varphi(\zeta) \\
& \lim _{z \rightarrow \zeta} \operatorname{Im}\{\overline{\lambda(\zeta)} \cdot f(z)\}=\psi(\zeta)
\end{aligned}
$$

along $\gamma_{\zeta}$ for a.e. $\zeta \in \partial D$ with respect to the logarithmic capacity. 
Remark 1. Thus, the space of all solutions $f$ of the Hilbert problem (5.2) in the given sense has the infinite dimension for any such prescribed $\varphi$, $\lambda$ and $\left\{\gamma_{\zeta}\right\}_{\zeta \in D}$ because the space of all functions $\psi: \partial D \rightarrow \mathbb{R}$ which are measurable with respect to the logarithmic capacity has the infinite dimension.

The latter is valid even for its subspace of continuous functions $\psi$ : $\partial D \rightarrow \mathbb{R}$. Indeed, by the Riemann theorem every Jordan domain $G$ can be mapped with a conformal mapping $g$ onto the unit disk $\mathbb{D}$ and by the Caratheodory theorem $g$ can be extended to a homeomorphism of $\bar{G}$ onto $\overline{\mathbb{D}}$. By the Fourier theory, the space of all continuous functions $\tilde{\psi}$ : $\partial \mathbb{D} \rightarrow \mathbb{R}$, equivalently, the space of all continuous $2 \pi$-periodic functions $\psi_{*}: \mathbb{R} \rightarrow \mathbb{R}$, has the infinite dimension.

Proof. Indeed, set $\Psi(\zeta)=\varphi(\zeta)+i \cdot \psi(\zeta)$ and $\Phi(\zeta)=\lambda(\zeta) \cdot \Psi(\zeta)$ for all $\zeta \in \partial D$. Then by Theorem 1 there is a single-valued analytic function $f$ such that

$$
\lim _{z \rightarrow \zeta} f(z)=\Phi(\zeta)
$$

along $\gamma_{\zeta}$ for a.e. $\zeta \in \partial D$ with respect to the logarithmic capacity. Then also

$$
\lim _{z \rightarrow \zeta} \overline{\lambda(\zeta)} \cdot f(z)=\Psi(\zeta)
$$

along $\gamma_{\zeta}$ for a.e. $\zeta \in \partial D$ with respect to the logarithmic capacity.

\section{On Riemann-Hilbert problem for Beltrami equations}

Theorem 4. Let $D$ be a bounded domain in $\mathbb{C}$ whose boundary consists of a finite number of mutually disjoint Jordan curves, $\mu: D \rightarrow \mathbb{C}$ be a Lebesgue measurable function with $\|\mu\|_{\infty}<1$, and let $\lambda: \partial D \rightarrow \mathbb{C}$, $|\lambda(\zeta)| \equiv 1, \varphi: \partial D \rightarrow \mathbb{R}$ and $\psi: \partial D \rightarrow \mathbb{R}$ be functions that are measurable with respect to the logarithmic capacity.

Suppose that $\left\{\gamma_{\zeta}\right\}_{\zeta \in \partial D}$ is a family of Jordan arcs of class $\mathcal{B S}$ in $D$. Then the Beltrami equation (1.1) has a regular solution $f: D \rightarrow \mathbb{C}$ such that

$$
\begin{aligned}
& \lim _{z \rightarrow \zeta} \operatorname{Re}\{\overline{\lambda(\zeta)} \cdot f(z)\}=\varphi(\zeta) \\
& \lim _{z \rightarrow \zeta} \operatorname{Im}\{\overline{\lambda(\zeta)} \cdot f(z)\}=\psi(\zeta)
\end{aligned}
$$

along $\gamma_{\zeta}$ for a.e. $\zeta \in \partial D$ with respect to the logarithmic capacity.

Proof. Indeed, set $\Psi(\zeta)=\varphi(\zeta)+i \cdot \psi(\zeta)$ and $\Phi(\zeta)=\lambda(\zeta) \cdot \Psi(\zeta)$ for all $\zeta \in \partial D$. Then by Theorem 2 the Beltrami equation (1.1) has a regular 
solution $f: D \rightarrow \mathbb{C}$ such that

$$
\lim _{z \rightarrow \zeta} f(z)=\Phi(\zeta)
$$

along $\gamma_{\zeta}$ for a.e. $\zeta \in \partial D$ with respect to the logarithmic capacity. Then also

$$
\lim _{z \rightarrow \zeta} \overline{\lambda(\zeta)} \cdot f(z)=\Psi(\zeta)
$$

along $\gamma_{\zeta}$ for a.e. $\zeta \in \partial D$ with respect to the logarithmic capacity.

Remark 2. Thus, the space of all solutions $f$ of the Hilbert problem (6.1) for the Beltrami equation (1.1) in the given sense has the infinite dimension for any such prescribed $\varphi, \lambda$ and $\left\{\gamma_{\zeta}\right\}_{\zeta \in D}$ because the space of all functions $\psi: \partial D \rightarrow \mathbb{R}$ which are measurable with respect to the logarithmic capacity has the infinite dimension, see Remark 1.

\section{Neumann and Poincare problems for analytic functions}

As known the Neumann problem has no classical solutions generally speaking even for harmonic functions and smooth boundary data, see e.g. [20]. Here we study the Neumann problem and more general boundary value problems for analytic functions with measurable boundary data in a generalized form.

First of all recall a more general problem on directional derivatives for the harmonic functions in the unit disk $\mathbb{D}=\{z \in \mathbb{C}:|z|<1\}, z=x+i y$. The classic setting of the latter problem is to find a function $u: \mathbb{D} \rightarrow \mathbb{R}$ that is twice continuously differentiable, admits a continuous extension to the boundary of $\mathbb{D}$ together with its first partial derivatives, satisfies the Laplace equation

$$
\Delta u:=\frac{\partial^{2} u}{\partial x^{2}}+\frac{\partial^{2} u}{\partial y^{2}}=0 \quad \forall z \in \mathbb{D}
$$

and the boundary condition with a prescribed continuous date $\varphi: \partial \mathbb{D} \rightarrow$ $\mathbb{R}$ :

$$
\frac{\partial u}{\partial \nu}=\varphi(\zeta) \quad \forall \zeta \in \partial \mathbb{D}
$$

where $\frac{\partial u}{\partial \nu}$ denotes the derivative of $u$ at $\zeta$ in a direction $\nu=\nu(\zeta)$, $|\nu(\zeta)|=1$ :

$$
\frac{\partial u}{\partial \nu}:=\lim _{t \rightarrow 0} \frac{u(\zeta+t \cdot \nu)-u(\zeta)}{t}
$$


The Neumann problem is a special case of the above problem on directional derivatives with the boundary condition

$$
\frac{\partial u}{\partial n}=\varphi(\zeta) \quad \forall \zeta \in \partial \mathbb{D}
$$

where $n$ denotes the unit interior normal to $\partial \mathbb{D}$ at the point $\zeta$.

In turn, the above problem on directional derivatives is a special case of the Poincare problem with the boundary condition

$$
a \cdot u+b \cdot \frac{\partial u}{\partial \nu}=\varphi(\zeta) \quad \forall \zeta \in \partial \mathbb{D}
$$

where $a=a(\zeta)$ and $b=b(\zeta)$ are real-valued functions given on $\partial \mathbb{D}$.

Recall also that twice continuously differentiable solutions of the Laplace equation are called harmonic functions. As known, such functions are infinitely differentiable as well as they are real and imaginary parts of analytic functions. The above boundary value problems for analytic functions are formulated in a similar way. Let us start from the problem on directional derivatives.

Theorem 5. Let $D$ be a Jordan domain in $\mathbb{C}$ with a rectifiable boundary, $\nu: \partial D \rightarrow \mathbb{C},|\nu(\zeta)| \equiv 1$, and $\Phi: \partial D \rightarrow \mathbb{C}$ be measurable functions with respect to the natural parameter.

Suppose that $\left\{\gamma_{\zeta}\right\}_{\zeta \in \partial D}$ is a family of Jordan arcs of class $\mathcal{B S}$ in D. Then there is a nonconstant single-valued analytic function $f: D \rightarrow \mathbb{C}$ such that

$$
\lim _{z \rightarrow \zeta} \frac{\partial f}{\partial \nu}(z)=\Phi(\zeta)
$$

along $\gamma_{\zeta}$ for a.e. $\zeta \in \partial D$ with respect to the natural parameter.

Proof. Indeed, by Theorem 5 in [4] there is a harmonic function $u: D \rightarrow$ $\mathbb{R}$ such that

$$
\lim _{z \rightarrow \zeta} \nabla u(z)=\Psi(\zeta):=\nu(\zeta) \cdot \overline{\Phi(\zeta)}
$$

along $\gamma_{\zeta}$ for a.e. $\zeta \in \partial D$ with respect to the natural parameter where we use the complex notation for the gradient $\nabla u=u_{x}+i \cdot u_{y}$.

As known, there is a conjugate harmonic function $v$ such that $f:=$ $u+i \cdot v$ is a single-valued analytic function in the simply connected domain $D$, see e.g. point I.A in [15]. The functions $u$ and $v$ satisfy the CauchyRiemann system $v_{x}=-u_{y}$ and $v_{y}=u_{x}$, i.e., $\nabla v=i \cdot \nabla u$. Note also that the derivatives of $u$ and $v$ in the direction $\nu$ are projections of gradients of $u$ and $v$ onto $\nu$. Hence

$$
\frac{\partial f}{\partial \nu}=\frac{\partial u}{\partial \nu}+i \cdot \frac{\partial v}{\partial \nu}=\operatorname{Re} \nu \cdot \overline{\nabla u}+i \cdot \operatorname{Re} \nu \cdot \overline{\nabla v}=\nu \cdot \overline{\nabla u} .
$$


Thus, (7.6) follows from (7.7) and, consequently, $f$ is the required function.

Remark 3. We are able to say more in the case $\operatorname{Re} n \cdot \bar{\nu}>0$ where $n=n(\zeta)$ is the unit interior normal with a tangent to $\partial D$ at a point $\zeta \in \partial D$. In view of (7.6), since the limit $\Phi(\zeta)$ is finite, there is a finite limit $f(\zeta)$ of $f(z)$ as $z \rightarrow \zeta$ in $D$ along the straight line passing through the point $\zeta$ and being parallel to the vector $\nu(\zeta)$ because along this line, for $z$ and $z_{0}$ that are close enough to $\zeta$,

$$
f(z)=f\left(z_{0}\right)-\int_{0}^{1} \frac{\partial f}{\partial \nu}\left(z_{0}+\tau\left(z-z_{0}\right)\right) d \tau .
$$

Thus, at each point with the condition (7.6), there is the directional derivative

$$
\frac{\partial f}{\partial \nu}(\zeta):=\lim _{t \rightarrow 0} \frac{f(\zeta+t \cdot \nu)-f(\zeta)}{t}=\Phi(\zeta) .
$$

In particular, the following statement on the solvability of the Neumann problem is a consequence of Theorem 5 and Remark 3.

Corollary 4. For each measurable function $\Phi: \partial \mathbb{D} \rightarrow \mathbb{C}$, one can find a nonconstant single-valued analytic function $f: \mathbb{D} \rightarrow \mathbb{C}$ such that, for a.e. point $\zeta \in \partial \mathbb{D}$, there exist:

1) the finite radial limit

$$
f(\zeta):=\lim _{r \rightarrow 1} f(r \zeta)
$$

2) the normal derivative

$$
\frac{\partial f}{\partial n}(\zeta):=\lim _{t \rightarrow 0} \frac{f(\zeta+t \cdot n)-f(\zeta)}{t}=\Phi(\zeta)
$$

3) the radial limit

$$
\lim _{r \rightarrow 1} \frac{\partial f}{\partial n}(r \zeta)=\frac{\partial f}{\partial n}(\zeta)
$$

where $n=n(\zeta)$ denotes the unit interior normal to $\partial \mathbb{D}$ at the point $\zeta$.

Finally, arguing as in the proof of Theorem 5 and using Corollary 2 instead of Theorem 5 in [4], we obtain the following theorem that solves the problem on directional derivatives for analytic functions in arbitrary Jordan domains. 
Theorem 6. Let $D$ be a Jordan domain in $\mathbb{C}, \nu: \partial D \rightarrow \mathbb{C},|\nu(\zeta)| \equiv 1$, and $\Phi: \partial D \rightarrow \mathbb{C}$ be measurable functions with respect to the logarithmic capacity.

Suppose that $\left\{\gamma_{\zeta}\right\}_{\zeta \in \partial D}$ is a family of Jordan arcs of class $\mathcal{B S}$ in D. Then there is a nonconstant single-valued analytic function $f: D \rightarrow \mathbb{C}$ such that

$$
\lim _{z \rightarrow \zeta} \frac{\partial f}{\partial \nu}(z)=\Phi(\zeta)
$$

along $\gamma_{\zeta}$ for a.e. $\zeta \in \partial D$ with respect to the logarithmic capacity.

Remark 4. As it follows from the given scheme of the proof, Theorems 5 and 6 are valid for multiply connected domains bounded by a finite collection of mutually disjoint Jordan curves, however, the corresponding analytic function $f$ can be multivalent.

\section{Neumann, Poincare problems for Beltrami equations}

Theorem 7. Let $D$ be a Jordan domain in $\mathbb{C}, \mu: D \rightarrow \mathbb{C}$ be a function of the Hölder class $C^{\alpha}$ with $\alpha \in(0,1)$ and $|\mu(z)| \leq k<1, z \in D$, and let $\nu: \partial D \rightarrow \mathbb{C},|\nu(\zeta)| \equiv 1$, and $\Phi: \partial D \rightarrow \mathbb{C}$ be measurable with respect to the logarithmic capacity.

Suppose that $\left\{\gamma_{\zeta}\right\}_{\zeta \in \partial D}$ is a family of Jordan arcs of class $\mathcal{B S}$ in $D$. Then the Beltrami equation (1.1) has a regular solution $f: D \rightarrow \mathbb{C}$ of the class $C^{1+\alpha}$ such that

$$
\lim _{z \rightarrow \zeta} \frac{\partial f}{\partial \nu}(z)=\Phi(\zeta)
$$

along $\gamma_{\zeta}$ for a.e. $\zeta \in \partial D$ with respect to the logarithmic capacity.

Lemma 1. Let $S$ be a subspace of a metric space $(X, d)$ and let $h: S \rightarrow \mathbb{R}$ be a Hölder continuous function, namely, for some $C>0$ and $\alpha \in(0,1)$,

$$
\left|h\left(x_{1}\right)-h\left(x_{2}\right)\right| \leq C \cdot d^{\alpha}\left(x_{1}, x_{2}\right) \quad \forall x_{1}, x_{2} \in S .
$$

Then there is an extension $H: X \rightarrow \mathbb{R}$ of $h$ to $X$ such that

$$
\left|H\left(x_{1}\right)-H\left(x_{2}\right)\right| \leq C \cdot d^{\alpha}\left(x_{1}, x_{2}\right) \quad \forall x_{1}, x_{2} \in X .
$$

The same is valid for the functions $h: S \rightarrow \mathbb{C}$ as well as $h: S \rightarrow \mathbb{R}^{n}$.

Proof. Let us prove that $d_{*}\left(x_{1}, x_{2}\right):=d^{\alpha}\left(x_{1}, x_{2}\right),\left(x_{1}, x_{2}\right) \in(X \times X)$, is a distance on $X$. Indeed, by the triangle inequality for $d$ we have that

$$
d_{*}\left(x_{1}, x_{2}\right) \leq\left(d\left(x_{1}, x_{3}\right)+d\left(x_{3}, x_{2}\right)\right)^{\alpha} \quad \forall x_{1}, x_{2}, x_{3} \in X .
$$


Hence it remains to show that

$$
\varphi(t, \tau):=t^{\alpha}+\tau^{\alpha}-(t+\tau)^{\alpha} \geq 0 \quad \forall t, \tau \in \mathbb{R}^{+}, \alpha \in(0,1) .
$$

It sufficient for this to note that $\varphi(t, 0) \equiv 0$ and

$$
\frac{\partial \varphi}{\partial \tau}(t, \tau)=\alpha \cdot\left[\frac{1}{\tau^{1-\alpha}}-\frac{1}{(t+\tau)^{1-\alpha}}\right] \geq 0 .
$$

Thus, the conclusion of Lemma 1 follows from point 2.10.44 in [7] on the Lipschitz functions.

Lemma 2. Let $f: D \rightarrow \mathbb{C}$ be a smooth quasiconformal mapping satisfying the Beltrami equation (1.1) with a continuous complex coefficient $\mu$ in a domain $D \subseteq \mathbb{C}$. Then

$$
C\left(Z_{f}\right)=0=C\left(f\left(Z_{f}\right)\right),
$$

where $C(E)$ is the logarithmic capacity of $E \subseteq \mathbb{C}, J_{f}$ is the Jacobian of $f$, and

$$
Z_{f}=\left\{z \in \mathbb{C}: J_{f}(z)=0\right\} .
$$

Proof. First of all, note that $f$ satisfies (1.1) for all $z \in D$ because the functions $\mu(z), f_{z}$ and $f_{\bar{z}}$ are continuous, and by (1.1) the maximal distortion $M_{f}(z)=\left|f_{z}\right|+\left|f_{\bar{z}}\right|$ under $f$ at $z$ is equal to 0 at all points of $Z_{f}$. Note also that, in view of countable subadditivity of logarithmic capacity, with no loss of generality we may assume that $D$ is bounded and then $C(D)<\infty$.

Let us take an arbitrary $\varepsilon>0$. In view of continuity of $M_{f}(z)$, for every $z_{*} \in Z_{f}$, there is a disk $B_{z_{*}}$ centered at $z_{*}$ with a small enough radius such that $B_{z_{*}} \subset D$ and such that $\sup _{z \in B} M_{f}(z) \leq \varepsilon$. The union of all these disks gives an open neighborhood $U$ of $Z_{f}$ such that $\sup _{z \in U} M_{f}(z) \leq \varepsilon$. The logarithmic capacity of a set can be characterized as its transfinite diameter, see e.g. [8] and the point 110 in [22]. Hence

$$
C\left(f\left(Z_{f}\right)\right) \leq C(f(U)) \leq \varepsilon \cdot C(U) \leq \varepsilon \cdot C(D) .
$$

Thus, by arbitrariness of $\varepsilon>0$ we obtain the right equality in (8.4).

Note that the function $J_{f}(z)=\left|f_{z}\right|^{2}-\left|f_{\bar{z}}\right|^{2}$ is continuous and, consequently, the set $Z_{f}$ is closed in $D$, i.e. $Z_{f}$ and $f\left(Z_{f}\right)$ are sigma-compacta in $D$ and $D_{*}=f(D)$, correspondingly. Thus, the equality $C\left(f\left(Z_{f}\right)\right)=0$ implies the equality $C\left(Z_{f}\right)=0$ by the same characterization of logarithmic capacity because the inverse mapping $f^{-1}$ is quasiconformal and hence it is Hölder continuous on every compact set in the domain $D_{*}$, see e.g. Theorem II.4.3 in [18]. 
Proof of Theorem 7. By Lemma $1 \mu$ is extended to a Hölder continuous function $\mu_{*}: \mathbb{C} \rightarrow \mathbb{C}$. Hence also, for every $k_{*} \in(k, 1)$, there is a neighborhood $U$ of the closure of the domain $D$ such that $|\mu(z)|<k_{*}$. Let $D_{*}$ be a connected component of $U$ containing $\bar{D}$.

Now, there is a quasiconformal mapping $h: D_{*} \rightarrow \mathbb{C}$ a.e. satisfying the Beltrami equation (1.1) with the complex coefficient $\mu^{*}:=\left.\mu_{*}\right|_{D_{*}}$ in $D_{*}$, see e.g. Theorem V.B.3 in [1]. Note that the mapping $h$ has the Hölder continuous first partial derivatives in $D_{*}$ with the same order of the Hölder continuity as $\mu$, see e.g. [11] and also [12]. Thus, by Lemma 2

$$
C\left(Z_{h}\right)=0=C\left(h\left(Z_{h}\right)\right),
$$

where $C(E)$ is the logarithmic capacity of $E \subseteq \mathbb{C}, J_{h}$ is the Jacobian of $h$, and

$$
Z_{h}=\left\{z \in \mathbb{C}: J_{h}(z)=0\right\} .
$$

Moreover, setting $D^{*}=h(D)$ and $\Gamma_{\xi}=h\left(\gamma_{h^{-1}(\xi)}\right), \xi \in \partial D^{*}$, we see that $\left\{\Gamma_{\xi}\right\}_{\xi \in \partial D^{*}}$ is a family of Jordan $\operatorname{arcs}$ of class $\mathcal{B S}$ in $D^{*}$.

Next, setting

$$
h_{\nu}(z):=\lim _{t \rightarrow 0} \frac{h(z+t \cdot \nu)-h(z)}{t \cdot \nu},
$$

we see that the function $h_{\nu}(z)$ is continuous and hence measurable and by (8.7) also $h_{\nu}(z) \neq 0$ a.e. in $z \in D_{*}$ with respect to the logarithmic capacity.

The logarithmic capacity of a set coincides with its transfinite diameter, see e.g. [8] and the point 110 in [22]. Hence the mappings $h$ and $h^{-1}$ transform sets of logarithmic capacity zero on $\partial D$ into sets of logarithmic capacity zero on $\partial D^{*}$ and vice versa because these quasiconformal mappings are Hölder continuous on $\partial D$ and $\partial D^{*}$ correspondingly, see e.g. Theorem II.4.3 in [18].

Further, the functions $\mathcal{N}:=\nu \circ h^{-1}$ and $\varphi:=\left(\Phi / h_{\nu}\right) \circ h^{-1}$ are measurable with respect to logarithmic capacity. Indeed, measurable sets with respect to logarithmic capacity are transformed under the mappings $h$ and $h^{-1}$ into measurable sets with respect to logarithmic capacity because such a set can be represented as the union of a sigma-compactum and a set of logarithmic capacity zero and compacta under continuous mappings are transformed into compacta and compacta are measurable with respect to logarithmic capacity.

Finally, by Theorem 6 there is a nonconstant single-valued analytic function $F: D^{*} \rightarrow \mathbb{C}$ such that

$$
\lim _{w \rightarrow \xi} \frac{\partial F}{\partial \mathcal{N}}(w)=\varphi(\xi)
$$


holds along $\Gamma_{\xi}$ for a.e. $\xi \in \partial D^{*}$ with respect to the logarithmic capacity.

It remains to note that $f:=F \circ h$ is the desired solution of (1.1) because

$$
\frac{\partial f}{\partial \nu}=F^{\prime} \circ h \cdot \frac{\partial h}{\partial \nu}=F^{\prime} \circ h \cdot \nu h_{\nu}=\frac{\partial F}{\partial \mathcal{N}} \circ h \cdot h_{\nu},
$$

i.e. (8.1) holds along $\gamma_{\zeta}$ for a.e. $\zeta \in \partial D$ with respect to the logarithmic capacity.

Remark 5. Again, we are able to say more in the case $\operatorname{Re} n \cdot \bar{\nu}>0$ where $n=n(\zeta)$ is the unit interior normal with a tangent to $\partial D$ at a point $\zeta \in \partial D$. In view of (8.1), since the limit $\Phi(\zeta)$ is finite, there is a finite limit $f(\zeta)$ of $f(z)$ as $z \rightarrow \zeta$ in $D$ along the straight line passing through the point $\zeta$ and being parallel to the vector $\nu(\zeta)$ because along this line, for $z$ and $z_{0}$ that are close enough to $\zeta$,

$$
f(z)=f\left(z_{0}\right)-\int_{0}^{1} \frac{\partial f}{\partial \nu}\left(z_{0}+\tau\left(z-z_{0}\right)\right) d \tau .
$$

Thus, at each point with the condition (8.1), there is the directional derivative

$$
\frac{\partial f}{\partial \nu}(\zeta):=\lim _{t \rightarrow 0} \frac{f(\zeta+t \cdot \nu)-f(\zeta)}{t}=\Phi(\zeta) .
$$

In particular, we obtain on the basis of Theorem 7 and Remark 5 the following statement on the Neumann problem.

Corollary 5. Let $\mu: \mathbb{D} \rightarrow \mathbb{C}$ be in $C^{\alpha}, \alpha \in(0,1)$, and $|\mu(z)| \leq k<1$, and let $\Phi: \partial \mathbb{D} \rightarrow \mathbb{C}$ be measurable with respect to the logarithmic capacity.

Then the Beltrami equation (1.1) has a regular solution $f: \mathbb{D} \rightarrow \mathbb{C}$ of the class $C^{1+\alpha}$ such that, for a.e. point $\zeta \in \partial \mathbb{D}$, there exist:

1) the finite radial limit

$$
f(\zeta):=\lim _{r \rightarrow 1} f(r \zeta)
$$

2) the normal derivative

$$
\frac{\partial f}{\partial n}(\zeta):=\lim _{t \rightarrow 0} \frac{f(\zeta+t \cdot n)-f(\zeta)}{t}=\Phi(\zeta)
$$

3) the radial limit

$$
\lim _{r \rightarrow 1} \frac{\partial f}{\partial n}(r \zeta)=\frac{\partial f}{\partial n}(\zeta)
$$

where $n=n(\zeta)$ denotes the unit interior normal to $\partial \mathbb{D}$ at the point $\zeta$. 
Remark 6. As it follows from Remark 4, Theorems 7 is valid for multiply connected domains $D$ bounded by a finite collection of mutually disjoint Jordan curves, however, then $f$ can be multivalent in the spirit of the theory of multivalent analytic functions.

Namely, in finitely connected domains $D$ in $\mathbb{C}$, we say that a continuous discrete open mapping $f: B\left(z_{0}, \varepsilon_{0}\right) \rightarrow \mathbb{C}$, where $B\left(z_{0}, \varepsilon_{0}\right) \subseteq D$, is a local regular solution of (1.1) if $f \in W_{\mathrm{loc}}^{1,1}, J_{f}(z) \neq 0$ and $f$ satisfies (1.1) a.e. in $B\left(z_{0}, \varepsilon_{0}\right)$. Local regular solutions $f_{0}: B\left(z_{0}, \varepsilon_{0}\right) \rightarrow \mathbb{C}$ and $f_{*}: B\left(z_{*}, \varepsilon_{*}\right) \rightarrow \mathbb{C}$ of $(1.1)$ are called the extension of each to other if there is a finite chain of such solutions $f_{i}: B\left(z_{i}, \varepsilon_{i}\right) \rightarrow \mathbb{C}$, $i=1, \ldots, m$, that $f_{1}=f_{0}, f_{m}=f_{*}$ and $f_{i}(z) \equiv f_{i+1}(z)$ for $z \in E_{i}:=$ $B\left(z_{i}, \varepsilon_{i}\right) \cap B\left(z_{i+1}, \varepsilon_{i+1}\right) \neq \emptyset, i=1, \ldots, m-1$. A collection of local regular solutions $f_{j}: B\left(z_{j}, \varepsilon_{j}\right) \rightarrow \mathbb{C}, j \in J$, will be called a multi-valued solution of the Beltrami equation (1.1) in $D$ if the disks $B\left(z_{j}, \varepsilon_{j}\right)$ cover the whole domain $D$ and $f_{j}$ are extensions of each to other through the collection and this collection is maximal by inclusion, cf. e.g. [5] and [16].

\section{On the Riemann problem for analytic functions}

Recall that the classical setting of the Riemann problem in a smooth Jordan domain $D$ of the complex plane $\mathbb{C}$ is to find analytic functions $f^{+}: D \rightarrow \mathbb{C}$ and $f^{-}: \mathbb{C} \backslash \bar{D} \rightarrow \mathbb{C}$ that admit continuous extensions to $\partial D$ and satisfy the boundary condition

$$
f^{+}(\zeta)=A(\zeta) \cdot f^{-}(\zeta)+B(\zeta) \quad \forall \zeta \in \partial D
$$

with prescribed Hölder continuous functions $A: \partial D \rightarrow \mathbb{C}$ and $B: \partial D \rightarrow$ $\mathbb{C}$.

Recall also that the Riemann problem with shift in $D$ is to find analytic functions $f^{+}: D \rightarrow \mathbb{C}$ and $f^{-}: \mathbb{C} \backslash \bar{D} \rightarrow \mathbb{C}$ satisfying the condition

$$
f^{+}(\alpha(\zeta))=A(\zeta) \cdot f^{-}(\zeta)+B(\zeta) \quad \forall \zeta \in \partial D
$$

where $\alpha: \partial D \rightarrow \partial D$ was a one-to-one sense preserving correspondence having the non-vanishing Hölder continuous derivative with respect to the natural parameter on $\partial D$. The function $\alpha$ is called a shift function. The special case $A \equiv 1$ gives the so-called jump problem and $B \equiv 0$ gives the problem on gluing of analytic functions.

Theorem 8. Let $D$ be a domain in $\overline{\mathbb{C}}$ whose boundary consists of a finite number of mutually disjoint Jordan curves, $A: \partial D \rightarrow \mathbb{C}$ and $B: \partial D \rightarrow \mathbb{C}$ be functions that are measurable with respect to the logarithmic capacity. 
Suppose that $\left\{\gamma_{\zeta}^{+}\right\}_{\zeta \in \partial D}$ and $\left\{\gamma_{\zeta}^{-}\right\}_{\zeta \in \partial D}$ are families of Jordan arcs of class $\mathcal{B S}$ in $D$ and $\mathbb{C} \backslash \bar{D}$, correspondingly.

Then there exist single-valued analytic functions $f^{+}: D \rightarrow \mathbb{C}$ and $f^{-}: \overline{\mathbb{C}} \backslash \bar{D} \rightarrow \mathbb{C}$ that satisfy (9.1) for a.e. $\zeta \in \partial D$ with respect to the logarithmic capacity where $f^{+}(\zeta)$ and $f^{-}(\zeta)$ are limits of $f^{+}(z)$ and $f^{-}(z)$ az $z \rightarrow \zeta$ along $\gamma_{\zeta}^{+}$and $\gamma_{\zeta}^{-}$, correspondingly.

Furthermore, the space of all such couples $\left(f^{+}, f^{-}\right)$has the infinite dimension for every couple $(A, B)$ and any collections $\gamma_{\zeta}^{+}$and $\gamma_{\zeta}^{-}, \zeta \in$ $\partial D$.

Theorem 8 is a special case of the following lemma on the generalized Riemann problem with shift that may have of independent interest.

Lemma 3. Under the hypotheses of Theorem 8, let in addition $\alpha: \partial D \rightarrow$ $\partial D$ be a homeomorphism keeping components of $\partial D$ such that $\alpha$ and $\alpha^{-1}$ have the $(N)$-property of Lusin with respect to the logarithmic capacity.

Then there exist single-valued analytic functions $f^{+}: D \rightarrow \mathbb{C}$ and $f^{-}: \overline{\mathbb{C}} \backslash \bar{D} \rightarrow \mathbb{C}$ that satisfy (9.2) for a.e. $\zeta \in \partial D$ with respect to the logarithmic capacity where $f^{+}(\zeta)$ and $f^{-}(\zeta)$ are limits of $f^{+}(z)$ and $f^{-}(z)$ az $z \rightarrow \zeta$ along $\gamma_{\zeta}^{+}$and $\gamma_{\zeta}^{-}$, correspondingly.

Furthermore, the space of all such couples $\left(f^{+}, f^{-}\right)$has the infinite dimension for every couple $(A, B)$ and any collections $\gamma_{\zeta}^{+}$and $\gamma_{\zeta}^{-}, \zeta \in$ $\partial D$.

Proof. For brevity, we write here " $C$-measurable" instead of the expression "measurable with respect to the logarithmic capacity".

First, let $D$ be bounded and let $g^{-}: \partial D \rightarrow \mathbb{C}$ be a $C$-measurable function. Then the function

$$
g^{+}:=\left\{A \cdot g^{-}+B\right\} \circ \alpha^{-1}
$$

is also $C$-measurable. Indeed, $E:=\left\{A \cdot g^{-}+B\right\}^{-1}(\Omega)$ is a subset of $\partial D$ that is $C$-measurable for every open set $\Omega \subseteq \mathbb{C}$ because the function $A \cdot g^{-}+B$ is $C$-measurable by the hypotheses. Hence the set $E$ is the union of a sigma-compact set and a set of the logarithmic capacity zero, see e.g. Section 2 in [6]. However, continuous mappings transform compact sets into compact sets and, thus, by $(N)$-property $\alpha(E)=$ $\alpha \circ\left\{A \cdot g^{-}+B\right\}^{-1}(\Omega)=\left(g^{+}\right)^{-1}(\Omega)$ is a $C$-measurable set, i.e. the function $g^{+}$is really $C$-measurable.

Then by Theorem 1 there is a single-valued analytic function $f^{+}$: $D \rightarrow \mathbb{C}$ such that

$$
\lim _{z \rightarrow \xi} f^{+}(z)=g^{+}(\xi)
$$


along $\gamma_{\xi}^{+}$for a.e. $\xi \in \partial D$ with respect to the logarithmic capacity. Note that $g^{+}(\alpha(\zeta))$ is determined by the given limit for a.e. $\zeta \in \partial D$ because $\alpha^{-1}$ also has the $(N)$-property of Lusin.

Note that $\overline{\mathbb{C}} \backslash \bar{D}$ consists of a finite number of (simply connected) Jordan domains $D_{0}, D_{1}, \ldots, D_{m}$ in the extended complex plane $\overline{\mathbb{C}}=$ $\mathbb{C} \cup\{\infty\}$. Let $\infty \in D_{0}$. Then again by Theorem 1 there exist singlevalued analytic functions $f_{l}^{-}: D_{l} \rightarrow \mathbb{C}, l=1, \ldots, m$, such that

$$
\lim _{z \rightarrow \zeta} f_{l}^{-}(z)=g_{l}^{-}(\zeta), \quad g_{l}^{-}:=\left.g^{-}\right|_{\partial D_{l}},
$$

along $\gamma_{\zeta}^{-}$for a.e. $\zeta \in \partial D_{l}$ with respect to the logarithmic capacity.

Now, let $S$ be a circle that contains $D$ and let $j$ be the inversion of $\overline{\mathbb{C}}$ with respect to $S$. Set

$$
D_{*}=j\left(D_{0}\right), g_{*}=\overline{g_{0} \circ j}, g_{0}^{-}:=\left.g^{-}\right|_{\partial D_{0}}, \gamma_{\xi}^{*}=j\left(\gamma_{j(\xi)}^{-}\right), \xi \in \partial D_{*} .
$$

Then by Theorem 1 there is a single-valued analytic function $f_{*}: D_{*} \rightarrow \mathbb{C}$ such that

$$
\lim _{w \rightarrow \xi} f_{*}(w)=g_{*}(\xi)
$$

along $\gamma_{\xi}^{*}$ for a.e. $\xi \in \partial D_{*}$ with respect to the logarithmic capacity. Note that $f_{0}^{-}:=\overline{g_{*} \circ j}$ is a single-valued analytic function in $D_{0}$ and by construction

$$
\lim _{z \rightarrow \zeta} f_{0}^{-}(z)=g_{0}^{-}(\zeta), \quad g_{0}^{-}:=\left.g^{-}\right|_{\partial D_{0}},
$$

along $\gamma_{\zeta}^{-}$for a.e. $\zeta \in \partial D_{0}$ with respect to the logarithmic capacity.

Thus, the functions $f_{l}^{-}, l=0,1, \ldots, m$, form an analytic function $f^{-}: \overline{\mathbb{C}} \backslash \bar{D} \rightarrow \mathbb{C}$ satisfying (9.2) for a.e. $\zeta \in \partial D$ with respect to the logarithmic capacity.

The space of all such couples $\left(f^{+}, f^{-}\right)$has the infinite dimension for every couple $(A, B)$ and any collections $\gamma_{\zeta}^{+}$and $\gamma_{\zeta}^{-}, \zeta \in \partial D$, in view of the above construction because of the space of all measurable functions $g^{-}: \partial D \rightarrow \mathbb{C}$ has the infinite dimension.

The case of unbounded $D$ is reduced to the case of bounded $D$ through the complex conjugation and the inversion of $\overline{\mathbb{C}}$ with respect to a circle $S$ in some of the components of $\overline{\mathbb{C}} \backslash \bar{D}$ arguing as above.

Remark 7. Some investigations were devoted also to the nonlinear Riemann problems with boundary conditions of the form

$$
\Phi\left(\zeta, f^{+}(\zeta), f^{-}(\zeta)\right)=0 \quad \forall \zeta \in \partial D
$$


It is natural as above to weaken such conditions to the following

$$
\Phi\left(\zeta, f^{+}(\zeta), f^{-}(\zeta)\right)=0 \quad \text { for a.e. } \quad \zeta \in \partial D
$$

It is easy to see that the proposed approach makes possible also to reduce such problems to the algebraic and measurable solvability of the relations

$$
\Phi(\zeta, v, w)=0
$$

with respect to complex-valued functions $v(\zeta)$ and $w(\zeta)$, cf. e.g. [10].

Example 1. For instance, correspondingly to the scheme given above, special nonlinear problems of the form

$$
f^{+}(\zeta)=\varphi\left(\zeta, f^{-}(\zeta)\right) \quad \text { for a.e. } \zeta \in \partial D
$$

are always solved if the function $\varphi: \partial D \times \mathbb{C} \rightarrow \mathbb{C}$ satisfies the Caratheodory conditions with respect to the logarithmic capacity: $\varphi(\zeta, w)$ is continuous in the variable $w \in \mathbb{C}$ for a.e. $\zeta \in \partial D$ with respect to the logarithmic capacity and it is $C$-measurable in the variable $\zeta \in \partial D$ for all $w \in \mathbb{C}$.

Furthermore, the spaces of solutions of such problems always have the infinite dimension. Indeed, by the Egorov theorem, see e.g. Theorem 2.3.7 in [7], see also Section 17.1 in [17], the function $\varphi(\zeta, \psi(\zeta))$ is $C$ measurable in $\zeta \in \partial D$ for every $C$-measurable function $\psi: \partial D \rightarrow \mathbb{C}$ if the function $\varphi$ satisfies the Caratheodory conditions, and the space of all $C$-measurable functions $\psi: \partial D \rightarrow \mathbb{C}$ has the infinite dimension, see e.g. Remark 1.

\section{On the Riemann problem for the Beltrami equations}

Theorem 9. Let $D$ be a domain in $\overline{\mathbb{C}}$ whose boundary consists of a finite number of mutually disjoint Jordan curves, $\mu: \mathbb{C} \rightarrow \mathbb{C}$ be a measurable (by Lebesgue) function with $\|\mu\|_{\infty}<1, A: \partial D \rightarrow \mathbb{C}$ and $B: \partial D \rightarrow \mathbb{C}$ be functions that are measurable with respect to the logarithmic capacity. Suppose that $\left\{\gamma_{\zeta}^{+}\right\}_{\zeta \in \partial D}$ and $\left\{\gamma_{\zeta}^{-}\right\}_{\zeta \in \partial D}$ are families of Jordan arcs of class $\mathcal{B S}$ in $D$ and $\mathbb{C} \backslash \bar{D}$, correspondingly.

Then the Beltrami equation (1.1) has regular solutions $f^{+}: D \rightarrow \mathbb{C}$ and $f^{-}: \overline{\mathbb{C}} \backslash \bar{D} \rightarrow \mathbb{C}$ that satisfy (9.1) for a.e. $\zeta \in \partial D$ with respect to the logarithmic capacity where $f^{+}(\zeta)$ and $f^{-}(\zeta)$ are limits of $f^{+}(z)$ and $f^{-}(z)$ az $z \rightarrow \zeta$ along $\gamma_{\zeta}^{+}$and $\gamma_{\zeta}^{-}$, correspondingly.

Furthermore, the space of all such couples $\left(f^{+}, f^{-}\right)$has the infinite dimension for every couple $(A, B)$ and any collections $\gamma_{\zeta}^{+}$and $\gamma_{\zeta}^{-}, \zeta \in$ $\partial D$. 
Theorem 9 is a special case of the following lemma on the Riemann problem with shift for the Beltrami equation.

Lemma 4. Under the hypotheses of Theorem 9, let in addition $\alpha: \partial D \rightarrow$ $\partial D$ be a homeomorphism keeping components of $\partial D$ such that $\alpha$ and $\alpha^{-1}$ have the $(N)$-property of Lusin with respect to the logarithmic capacity.

Then the Beltrami equation (1.1) has regular solutions $f^{+}: D \rightarrow \mathbb{C}$ and $f^{-}: \overline{\mathbb{C}} \backslash \bar{D} \rightarrow \mathbb{C}$ that satisfy (9.2) for a.e. $\zeta \in \partial D$ with respect to the logarithmic capacity where $f^{+}(\zeta)$ and $f^{-}(\zeta)$ are limits of $f^{+}(z)$ and $f^{-}(z)$ az $z \rightarrow \zeta$ along $\gamma_{\zeta}^{+}$and $\gamma_{\zeta}^{-}$, correspondingly.

Furthermore, the space of all such couples $\left(f^{+}, f^{-}\right)$has the infinite dimension for every couple $(A, B)$ and any collections $\gamma_{\zeta}^{+}$and $\gamma_{\zeta}^{-}, \zeta \in$ $\partial D$.

Proof. First of all, there is a quasiconformal mapping $h: \mathbb{C} \rightarrow \mathbb{C}$ that is a regular homeomorphic solution of the Beltrami equation (1.1) with the given complex coefficient $\mu$, see e.g. Theorem V.B.3 in [1]. Set $D^{*}=$ $h(D)$ and $\alpha_{*}=h \circ \alpha \circ h^{-1}$. Then $\alpha_{*}: \partial D_{*} \rightarrow \partial D_{*}$ is a homeomorphism.

Recall that the logarithmic capacity of a set coincides with its transfinite diameter, see [8] and the point 110 in [22]. Hence the mappings $h$ and $h^{-1}$ transform sets of logarithmic capacity zero on $\partial D$ into sets of logarithmic capacity zero on $\partial D^{*}$ and vice versa because quasiconformal mappings are continuous by Hölder on $\partial D$ and $\partial D^{*}$ correspondingly, see e.g. Theorem II.4.3 in [18]. Thus, $\alpha_{*}$ and $\alpha_{*}^{-1}$ have the $(N)$-property with respect to the logarithmic capacity.

Moreover, arguing similarly to the proof of Theorem 7, it is easy to show that the functions $A_{*}:=A \circ h^{-1}$ and $B_{*}:=B \circ h^{-1}$ are measurable with respect to the logarithmic capacity. Setting $\Gamma_{\xi}^{+}=h\left(\gamma_{h^{-1}(\xi)}^{+}\right)$and $\Gamma_{\xi}^{-}=h\left(\gamma_{h^{-1}(\xi)}^{-}\right), \xi \in \partial D^{*}$, we see also that $\left\{\Gamma_{\xi}^{+}\right\}_{\xi \in \partial D^{*}}$ and $\left\{\Gamma_{\xi}^{-}\right\}_{\xi \in \partial D^{*}}$ are families of Jordan arcs of class $\mathcal{B S}$ in $D^{*}$ and $\mathbb{C} \backslash \overline{D^{*}}$, correspondingly.

Hence by Lemma 3 there exist single-valued analytic functions $f_{*}^{+}$: $D_{*} \rightarrow \mathbb{C}$ and $f_{*}^{-}: \overline{\mathbb{C}} \backslash \overline{D_{*}} \rightarrow \mathbb{C}$ such that

$$
f_{*}^{+}\left(\alpha_{*}(\xi)\right)=A_{*}(\xi) \cdot f_{*}^{-}(\xi)+B_{*}(\xi) \quad \forall \xi \in \partial D_{*}
$$

for a.e. $\xi \in \partial D_{*}$ with respect to the logarithmic capacity where $f_{*}^{+}(\xi)$ and $f_{*}^{-}(\xi)$ are limits of $f_{*}^{+}(w)$ and $f_{*}^{-}(w)$ as $w \rightarrow \xi$ along $\Gamma_{\xi}^{+}$and $\Gamma_{\xi}^{-}$. Furthermore, the space of all such couples $\left(f_{*}^{+}, f_{*}^{-}\right)$has the infinite dimension.

Finally, $f^{+}:=f_{*}^{+} \circ h$ and $f^{-}:=f_{*}^{-} \circ h$ are the desired regular solutions of the Beltrami equation (1.1) in $D$ and $\mathbb{C} \backslash \bar{D}$, correspondingly. 
Remark 8. As it follows from the construction of solutions in the given proof, Remark 6 on the nonlinear Riemann problems is valid for the Beltrami equations, see also Example 1 in the last section.

\section{On mixed boundary value problems}

In order to demonstrate the potentiality of our approach, we consider here some nonlinear boundary value problems.

Theorem 10. Let $D$ be a domain in $\overline{\mathbb{C}}$ whose boundary consists of a finite number of mutually disjoint Jordan curves, $\mu: \mathbb{C} \rightarrow \mathbb{C}$ be Lebesgue measurable, $\mu \in C^{\alpha}(\mathbb{C} \backslash \bar{D})$ and $\|\mu\|_{\infty}<1, \varphi: \partial D \times \mathbb{C} \rightarrow \mathbb{C}$ satisfy the Carathéodory conditions and $\nu: \partial D \rightarrow \mathbb{C},|\nu(\zeta)| \equiv 1$, be measurable with respect to the logarithmic capacity.

Suppose that $\left\{\gamma_{\zeta}^{+}\right\}_{\zeta \in \partial D}$ and $\left\{\gamma_{\zeta}^{-}\right\}_{\zeta \in \partial D}$ are families of Jordan arcs of class $\mathcal{B S}$ in $D$ and $\mathbb{C} \backslash \bar{D}$. Then the Beltrami equation (1.1) has regular solutions $f^{+}: D \rightarrow \mathbb{C}$ and $f^{-}: \overline{\mathbb{C}} \backslash \bar{D} \rightarrow \mathbb{C}$ of class $C^{1+\alpha}(\mathbb{C} \backslash \bar{D})$ such that

$$
f^{+}(\zeta)=\varphi\left(\zeta,\left[\frac{\partial f}{\partial \nu}\right]^{-}(\zeta)\right)
$$

for a.e. $\zeta \in \partial D$ with respect to the logarithmic capacity where $f^{+}(\zeta)$ and $\left[\frac{\partial f}{\partial \nu}\right]^{-}(\zeta)$ are limits of the functions $f^{+}(z)$ and $\frac{\partial f^{-}}{\partial \nu}(z)$ as $z \rightarrow \zeta$ along $\gamma_{\zeta}^{+}$and $\gamma_{\zeta}^{-}$, correspondingly.

Furthermore, the space of all such couples $\left(f^{+}, f^{-}\right)$has the infinite dimension for any such prescribed functions $\mu, \varphi, \nu$ and collections $\gamma_{\zeta}^{+}$ and $\gamma_{\zeta}^{-}, \zeta \in \partial D$.

Theorem 10 is a special case of the following lemma on the nonlinear Riemann problem with shift.

Lemma 5. Under the hypotheses of Theorem 10, let in addition $\beta$ : $\partial D \rightarrow \partial D$ be a homeomorphism keeping components of $\partial D$ such that $\beta$ and $\beta^{-1}$ have the $(N)$-property of Lusin with respect to the logarithmic capacity.

Then the Beltrami equation (1.1) has regular solutions $f^{+}: D \rightarrow \mathbb{C}$ and $f^{-}: \overline{\mathbb{C}} \backslash \bar{D} \rightarrow \mathbb{C}$ of class $C^{1+\alpha}(\mathbb{C} \backslash \bar{D})$ such that

$$
f^{+}(\beta(\zeta))=\varphi\left(\zeta,\left[\frac{\partial f}{\partial \nu}\right]^{-}(\zeta)\right)
$$


for a.e. $\zeta \in \partial D$ with respect to the logarithmic capacity where $f^{+}(\zeta)$ and $\left[\frac{\partial f}{\partial \nu}\right]^{-}(\zeta)$ are limits of the functions $f^{+}(z)$ and $\frac{\partial f^{-}}{\partial \nu}(z)$ as $z \rightarrow \zeta$ along $\gamma_{\zeta}^{+}$and $\gamma_{\zeta}^{-}$, correspondingly.

Furthermore, the space of all such couples $\left(f^{+}, f^{-}\right)$has the infinite dimension for any such prescribed $\mu, \varphi, \nu, \beta$ and collections $\left\{\gamma_{\zeta}^{+}\right\}_{\zeta \in \partial D}$ and $\left\{\gamma_{\zeta}^{-}\right\}_{\zeta \in \partial D}$.

Proof. Indeed, let $\psi: \partial D \rightarrow \mathbb{C}$ be a function that is measurable with respect to the logarithmic capacity. Then by Theorem 7 the Beltrami equation (1.1) has a regular solution $f^{-}: \overline{\mathbb{C}} \backslash \bar{D} \rightarrow \mathbb{C}$ of class $C^{1+\alpha}$ such that

$$
\lim _{z \rightarrow \zeta} \frac{\partial f^{-}}{\partial \nu}(z)=\psi(\zeta)
$$

along $\gamma_{\zeta}^{-}$for a.e. $\zeta \in \partial D$ with respect to the logarithmic capacity.

Now, the function $\Psi(\zeta):=\varphi(\zeta, \psi(\zeta))$ is measurable with respect to the logarithmic capacity, see Example 1 after Remark 6 . Then the function $\Phi=\Psi \circ \alpha^{-1}$ is also measurable with respect to the logarithmic capacity because the homeomorphism $\alpha$ has the $(N)$-property, cf. arguments in the proof of Lemma 4.

Thus, by Theorem 2 the Beltrami equation (1.1) has a regular solution $f^{+}: D \rightarrow \mathbb{C}$ such that $f^{+}(z) \rightarrow \Psi(\zeta)$ as $z \rightarrow \zeta$ along $\gamma_{\zeta}$ for a.e. $\zeta \in \partial D$ with respect to the logarithmic capacity. Finally, $f^{+}$and $f^{-}$are the desired functions because $\alpha^{-1}$ also has the $(N)$-property.

It remains also to note that the space of all such couples $\left(f^{+}, f^{-}\right)$ has the infinite dimension because the space of all functions $\psi: \partial D \rightarrow \mathbb{C}$ which are measurable with respect to the logarithmic capacity has the infinite dimension, see Remark 1.

\section{On applications to equations of the divergence type}

The partial differential equations in the divergence form below take a significant part in many problems of mathematical physics, in particular, in anisotropic inhomogeneous media, see e.g. [3,13] and [14].

In this connection, note that if $f=u+i \cdot v$ is a regular solution of the Beltrami equation (1.1), then the function $u$ is a continuous generalized solution of the divergence-type equation

$$
\operatorname{div} A(z) \nabla u=0,
$$

called $A$-harmonic function, i.e. $u \in C \cap W^{1,1}$ and

$$
\int_{D}\langle A(z) \nabla u, \nabla \varphi\rangle=0 \quad \forall \varphi \in C_{0}^{\infty}(D),
$$


where $A(z)$ is the matrix function:

$$
A=\left(\begin{array}{cc}
\frac{|1-\mu|^{2}}{1-|\mu|^{2}} & \frac{-2 \operatorname{Im} \mu}{1-|\mu|^{2}} \\
\frac{-2 \operatorname{Im} \mu}{1-|\mu|^{2}} & \frac{|1+\mu|^{2}}{1-|\mu|^{2}}
\end{array}\right)
$$

Moreover, we have for the stream function $v=\operatorname{Im} f$ the following relation

$$
\nabla v=J A(z) \nabla u
$$

where

$$
J=\left(\begin{array}{cc}
0 & -1 \\
1 & 0
\end{array}\right) .
$$

As we see in (12.3), the matrix $A(z)$ is symmetric and its entries $a_{i j}=$ $a_{i j}(z)$ are dominated by the quantity

$$
K_{\mu}(z)=\frac{1+|\mu(z)|}{1-|\mu(z)|},
$$

and, thus, they are bounded if the Beltrami equation (1.1) is not degenerate.

Vice verse, uniformly elliptic equations (12.1) with symmetric $A(z)$ and $\operatorname{det} A(z) \equiv 1$ just correspond to nondegenerate Beltrami equations (1.1). Let all such matrix functions $A(z)$ form the class $\mathcal{B}$.

Recall that the equation (12.1) is called uniformly elliptic if $a_{i j} \in L^{\infty}$ and there is $\varepsilon>0$ such that $\langle A(z) \eta, \eta\rangle \geq \varepsilon|\eta|^{2}$ for all $\eta \in \mathbb{R}^{2}$. A matrix function $A(z)$ with the latter property is called uniformly positive definite.

The following statement on a potential boundary behavior of Aharmonic functions of the Dirichlet type is a direct consequence of Theorem 2 .

Corollary 6. Let $D$ be a bounded domain in $\mathbb{C}$ whose boundary consists of a finite number of mutually disjoint Jordan curves, $A(z), z \in D$, be a matrix function of class $\mathcal{B}$ and let a function $\varphi: \partial D \rightarrow \mathbb{R}$ be measurable with respect to the logarithmic capacity.

Suppose that $\left\{\gamma_{\zeta}\right\}_{\zeta \in \partial D}$ is a family of Jordan arcs of class $\mathcal{B S}$ in $D$. Then there exist $A$-harmonic functions $u: D \rightarrow \mathbb{R}$ such that

$$
\lim _{z \rightarrow \zeta} u(z)=\varphi(\zeta)
$$

along $\gamma_{\zeta}$ for a.e. $\zeta \in \partial D$ with respect to the logarithmic capacity.

Furthermore, the space of all such A-harmonic functions $u$ has the infinite dimension for any such prescribed $A, \varphi$ and $\left\{\gamma_{\zeta}\right\}_{\zeta \in \partial D}$. 
The next conclusion in the particular case of the Poincare problem on directional derivatives follows directly from Theorem 7 .

Corollary 7. Let $D$ be a Jordan domain in $\mathbb{C}, A(z), z \in D$, be a matrix function of class $\mathcal{B} \cap C^{\alpha}, \alpha \in(0,1)$, and let $\nu: \partial D \rightarrow \mathbb{C},|\nu(\zeta)| \equiv 1$, and $\varphi: \partial D \rightarrow \mathbb{R}$ be measurable with respect to the logarithmic capacity.

Suppose that $\left\{\gamma_{\zeta}\right\}_{\zeta \in \partial D}$ is a family of Jordan arcs of class $\mathcal{B S}$ in D. Then there exist $A$-harmonic functions $u: D \rightarrow \mathbb{R}$ of the class $C^{1+\alpha}$ such that

$$
\lim _{z \rightarrow \zeta} \frac{\partial u}{\partial \nu}(z)=\varphi(\zeta)
$$

along $\gamma_{\zeta}$ for a.e. $\zeta \in \partial D$ with respect to the logarithmic capacity.

Furthermore, the space of all such A-harmonic functions $u$ has the infinite dimension for any such prescribed $A, \varphi, \nu$ and $\left\{\gamma_{\zeta}\right\}_{\zeta \in \partial D}$.

Now, the following statement concerning to the Neumann problem for A-harmonic functions is a special significant case of Corollary 7 .

Corollary 8. Let $A(z), z \in \mathbb{D}$, be a matrix function of class $\mathcal{B} \cap C^{\alpha}, \alpha \in$ $(0,1)$, and let $\varphi: \partial \mathbb{D} \rightarrow \mathbb{R}$ be measurable with respect to the logarithmic capacity.

Then there exist $A$-harmonic functions $u: \mathbb{D} \rightarrow \mathbb{R}$ of the class $C^{1+\alpha}$ such that, for a.e. point $\zeta \in \partial \mathbb{D}$ with respect to the logarithmic capacity, there exist:

1) the finite radial limit

$$
u(\zeta):=\lim _{r \rightarrow 1} u(r \zeta)
$$

2) the normal derivative

$$
\frac{\partial u}{\partial n}(\zeta):=\lim _{t \rightarrow 0} \frac{u(\zeta+t \cdot n)-u(\zeta)}{t}=\varphi(\zeta),
$$

3) the radial limit

$$
\lim _{r \rightarrow 1} \frac{\partial u}{\partial n}(r \zeta)=\frac{\partial u}{\partial n}(\zeta)
$$

where $n=n(\zeta)$ denotes the unit interior normal to $\partial \mathbb{D}$ at the point $\zeta$.

Furthermore, the space of all such A-harmonic functions $u$ has the infinite dimension for any such prescribed $A$ and $\varphi$.

The following result on the Riemann problem with shift for A-harmonic functions is not a direct consequence of Lemma 4 but of its proof where we are able to choose $f^{-}(\zeta)$ such that $\operatorname{Im} f^{-}(\zeta)=0$ for a.e. $\zeta \in \partial D$ with respect to the logarithmic capacity. 
Corollary 9. Let $D$ be a domain in $\mathbb{C}$ whose boundary consists of a finite number of mutually disjoint Jordan curves, $A(z), z \in D$, be a matrix function of class $\mathcal{B}, B: \partial D \rightarrow \mathbb{R}$ and $C: \partial D \rightarrow \mathbb{R}$ be functions that are measurable with respect to the logarithmic capacity and let $\alpha: \partial D \rightarrow \partial D$ be a homeomorphism keeping components of $\partial D$ such that $\alpha$ and $\alpha^{-1}$ have the $(N)$-property of Lusin with respect to the logarithmic capacity.

Suppose that $\left\{\gamma_{\zeta}^{+}\right\}_{\zeta \in \partial D}$ and $\left\{\gamma_{\zeta}^{-}\right\}_{\zeta \in \partial D}$ are families of Jordan arcs of class $\mathcal{B S}$ in $D$ and $\mathbb{C} \backslash \bar{D}$, correspondingly. Then there exist A-harmonic functions $u^{+}: D \rightarrow \mathbb{R}$ and $u^{-}: \overline{\mathbb{C}} \backslash \bar{D} \rightarrow \mathbb{R}$ such that

$$
u^{+}(\alpha(\zeta))=B(\zeta) \cdot u^{-}(\zeta)+C(\zeta)
$$

for a.e. $\zeta \in \partial D$ with respect to the logarithmic capacity where $u^{+}(\zeta)$ and $u^{-}(\zeta)$ are limits of $u^{+}(z)$ and $u^{-}(z)$ az $z \rightarrow \zeta$ along $\gamma_{\zeta}^{+}$and $\gamma_{\zeta}^{-}$, correspondingly.

Furthermore, the space of all such couples $\left(u^{+}, u^{-}\right)$has the infinite dimension for any such prescribed $A, B, C, \alpha$ and collections $\left\{\gamma_{\zeta}^{+}\right\}_{\zeta \in \partial D}$ and $\left\{\gamma_{\zeta}^{-}\right\}_{\zeta \in \partial D}$.

Similarly, the following result on the mixed nonlinear Riemann problem with shifts for A-harmonic functions is not a direct consequence of Lemma 5 but of its proof where we may choose $f^{-}(\zeta)$ such that $\operatorname{Im}\left[\frac{\partial f}{\partial \nu}\right]^{-}(\zeta)=0$ for a.e. $\zeta \in \partial D$ with respect to the logarithmic capacity.

Corollary 10. Let $D$ be a domain in $\mathbb{C}$ whose boundary consists of a finite number of mutually disjoint Jordan curves, $A(z), z \in \mathbb{C}$, be a matrix function of class $\mathcal{B} \cap C^{\alpha}(\mathbb{C} \backslash \bar{D}), \alpha \in(0,1), \nu: \partial D \rightarrow \mathbb{C},|\nu(\zeta)| \equiv 1$, be a measurable function, $\beta: \partial D \rightarrow \partial D$ be a homeomorphism such that $\beta$ and $\beta^{-1}$ have the $(N)$-property of Lusin and $\varphi: \partial D \times \mathbb{R} \rightarrow \mathbb{R}$ satisfy the Caratheodory conditions with respect to the logarithmic capacity.

Suppose that $\left\{\gamma_{\zeta}^{+}\right\}_{\zeta \in \partial D}$ and $\left\{\gamma_{\zeta}^{-}\right\}_{\zeta \in \partial D}$ are families of Jordan arcs of class $\mathcal{B S}$ in $D$ and $\mathbb{C} \backslash \bar{D}$, correspondingly. Then there exist A-harmonic functions $u^{+}: D \rightarrow \mathbb{R}$ and $u^{-}: \mathbb{C} \backslash \bar{D} \rightarrow \mathbb{R}$ of class $C^{1+\alpha}(\mathbb{C} \backslash \bar{D})$ such that

$$
u^{+}(\beta(\zeta))=\varphi\left(\zeta,\left[\frac{\partial u}{\partial \nu}\right]^{-}(\zeta)\right)
$$

for a.e. $\zeta \in \partial D$ with respect to the logarithmic capacity where $u^{+}(\zeta)$ and $\left[\frac{\partial u}{\partial \nu}\right]^{-}(\zeta)$ are limits of the functions $u^{+}(z)$ and $\frac{\partial u^{-}}{\partial \nu}(z)$ as $z \rightarrow \zeta$ along $\gamma_{\zeta}^{+}$and $\gamma_{\zeta}^{-}$, correspondingly.

Furthermore, the space of all such couples $\left(u^{+}, u^{-}\right)$has the infinite dimension for any such prescribed $A, \nu, \beta, \varphi$ and collections $\gamma_{\zeta}^{+}$and $\gamma_{\zeta}^{-}$, $\zeta \in \partial D$. 
In particular, we are able to obtain from the last corollary solutions of the problem on gluing of the Dirichlet problem in the unit disk $\mathbb{D}$ and the Neumann problem outside of $\mathbb{D}$ in the class of A-harmonic functions.

\section{References}

[1] L. Ahlfors, Lectures on Quasiconformal Mappings, Van Nostrand, New York, 1966.

[2] L. Ahlfors, A. Beurling, The boundary correspondence under quasiconformal mappings // Acta Math., 96 (1956), 125--142.

[3] K. Astala, T. Iwaniec, G. Martin, Elliptic partial differential equations and quasiconformal mappings in the plane // Princeton Math. Ser., 48, Princeton Univ. Press, Princeton, NJ, 2009.

[4] F. Bagemihl, W. Seidel, Regular functions with prescribed measurable boundary values almost everywhere // Proc. Nat. Acad. Sci. U. S. A. 41 (1955), 740-743.

[5] B. Bojarski, V. Gutlyanskii, V. Ryazanov, On existence and representation of solutions for general degenerate Beltrami equations // Complex Variables and Elliptic Equations, 59 (2014), No. 1, 67--75.

[6] A. Efimushkin, V. Ryazanov, On the Riemann-Hilbert problem for the Beltrami equations in quasidisks // Ukr. Math. Vis. 12 (2015), No. 2, 190-209; see also arXiv.org: 1404.1111v4 [math.CV] 22 Sep 2015, 1-25.

[7] H. Federer, Geometric Measure Theory, Springer-Verlag, Berlin, 1969.

[8] M. Fékete, Über die Verteilung der Wurzeln bei gewissen algebraischen Gleichungen mit ganzzahligen Koeffizienten // Math. Z., 17 (1923), 228-249.

[9] F. D. Gakhov, Boundary value problems, Dover Publications. Inc., New York, 1990.

[10] M. Gromov, Partial differential relations, Ergebnisse der Mathematik und ihrer Grenzgebiete (3) [Results in Mathematics and Related Areas (3)], 9. SpringerVerlag, Berlin, 1986.

[11] T. Iwaniec, Regularity of solutions of certain degenerate elliptic systems of equations that realize quasiconformal mappings in n-dimensional space // Differential and integral equations. Boundary value problems. Tbilisi: Tbilis. Gos. Univ., 1979, 97-111 (in Russian).

[12] T. Iwaniec, Regularity theorems for solutions of partial differential equations for quasiconformal mappings in several dimensions // Dissertationes Math. (Rozprawy Mat.) 198 (1982), 45.

[13] T. Iwaniec, p-harmonic tensors and quasiregular mappings // Ann. of Math., (2) 136 (1992), No. 3, 589-624.

[14] T. Iwaniec, C. Sbordone, Quasiharmonic fields // Ann. Inst. H. Poincare Anal. Non Lineaire, 18, (2001), No. 5, 519-572.

[15] P. Koosis, Introduction to $H_{p}$ spaces // Cambridge Tracts in Mathematics, 115, Cambridge Univ. Press, Cambridge, 1998.

[16] D. Kovtonyuk, I. Petkov, V. Ryazanov, R. Salimov, On the Dirichlet problem for the Beltrami equation // J. Anal. Math., 122 (2014), No. 4, 113-141.

[17] M. A. Krasnosel'skii, P. P. Zabreiko, E. I. Pustyl'nik, P. E. Sobolevskii, Integral operators in spaces of summable functions // Monographs and Textbooks on Mechanics of Solids and Fluids, Mechanics: Analysis, Noordhoff International Publishing, Leiden, 1976. 
[18] O. Lehto, K. J. Virtanen, Quasiconformal mappings in the plane, SpringerVerlag, Berlin-Heidelberg, 1973.

[19] O. Martio, S. Rickman, J. Vaisala, Definitions for quasiregular mappings // Ann. Acad. Sci. Fenn. Ser. A1. Math., 448 (1969), 1-40.

[20] S. G. Mikhlin, Partielle Differentialgleichungen in der mathematischen Physik // Math. Lehrbücher und Monographien, 30, Akademie-Verlag, Berlin, 1978.

[21] N. I. Muskhelishvili, Singular integral equations. Boundary problems of function theory and their application to mathematical physics, Dover Publications. Inc., New York, 1992.

[22] R. Nevanlinna, Eindeutige analytische Funktionen, Ann Arbor, Michigan, 1944.

[23] I. I. Priwalow, Randeigenschaften analytischer Funktionen. VEB Deutscher Verlag der Wissenschaften, Berlin, 1956.

[24] Yu. G. Reshetnyak, Space mappings with bounded distortion, Nauka, Novosibirsk, 1982; Transl. of Mathematical Monographs, 73, Amer. Math. Soc., Providence, RI, 1988.

[25] V. Ryazanov, On the Riemann-Hilbert Problem without Index // Ann. Univ. Bucharest, Ser. Math. 5 (LXIII) (2014), No. 1, 169-178.

[26] V. Ryazanov, Infinite dimension of solutions of the Dirichlet problem // Open Math. (the former Central European J. Math.), 13 (2015), No. 1, 348-350.

[27] V. Ryazanov, On Neumann and Poincare problems for Laplace equation // arXiv.org: 1510.00733v5 [math.CV] 26 Oct 2015, 1-5.

[28] V. Ryazanov, On Hilbert and Riemann problems. An alternative approach // arXiv.org: 1510.06380v3 [math.CV] 28 Oct 2015, 1-8.

[29] I. N. Vekua, Generalized analytic functions, Pergamon Press, London etc., 1962.

\section{CONTACT INFORMATION}

Vladimir

Gutlyanskii

Vladimir Ryazanov

Artem Yefimushkin
Institute of Applied Mathematics

and Mechanics, NAS of Ukraine

E-Mail: vladimirgut@mail.ru

vl.ryazanov1@gmail.com

a.yefimushkin@gmail.com 